\title{
A medicalização social e o uso do metilfenidato no aprimoramento cognitivo
} farmacológico ${ }^{1}$

The social medicalization and the use of methylphenidate in the pharmacological cognitive enhancement

La medicalización social y el uso de metilfenidato en el mejoramiento cognitivo farmacológico

Recebido: 07/05/2020 | Revisado: 07/05/2020 | Aceito: 09/05/2020 | Publicado: 17/05/2020

Emilia Suitberta de Oliveira Trigueiro ORCID: http://orcid.org/0000-0002-7649-7688 Centro Universitário Dr. Leão Sampaio, Brasil E-mail: emiliatrigueiro@ hotmail.com

\section{Resumo}

O uso de medicamentos, por pessoas saudáveis, para melhorar o funcionamento do cérebro e aprimorar o desempenho cognitivo pode ser chamado de aprimoramento cognitivo farmacológico ou de doping intelectual. Um dos medicamentos mais utilizados no Brasil com esta finalidade é o Cloridrato de Metilfenidato, comercializado com o nome de Ritalina ou Concerta. O presente artigo objetivou compreender, por meio de uma revisão de literatura, como o cloridrato metilfenidato tem sido utilizado no aprimoramento cognitivo farmacológico e a relação deste fenômeno com a medicalização social. Sabe-se que estes medicamentos tem como efeito imediato o aumento da concentração, mas a longo prazo podem causar supressão do crescimento, aumento da pressão sanguínea, episódios psicóticos, entre outros. Ainda assim, eles tem sido utilizados por estudantes universitários com a finalidade de aumentar sua capacidade produtiva e cumprir prazos e metas. Como esta prática tem ganhado cada vez mais adeptos e o acesso a este medicamento é facilitado, é necessário aprofundar o entendimento deste fenômeno.

Palavras-chave: Educação; Medicalização; Metilfenidato; Aprimoramento cognitivo farmacológico; Melhoramento cognitivo.

\footnotetext{
${ }^{1} \mathrm{O}$ presente artigo é fruto da tese de doutorado da autora.
} 


\begin{abstract}
The use of drugs by healthy people to improve the brain functioning and enhance the cognitive performance might be called as pharmacological cognitive enhancement or intellectual doping. In Brazil, one of the most common drugs used for this purpose is the Methylphenidate Hydrochloride, which is marketed as Ritalina (Ritalin) or Concerta. This article aimed to understand, through a literature review, how methylphenidate hydrochloride has been used in the pharmacological cognitive enhancement and the relationship of this phenomenon with social medicalization. It is known that these drugs have the immediate effect of increasing concentration, but in the long term, they may cause growth suppression, increasing the blood pressure, psychotic episodes, etc. Even so, they have been used by academics with the purpose of increasing their production capacity and meeting deadlines and targets. Considering the increasing of the fans of this pratice and the facilitation of access to this medicine, it is necessary to go deep into the understanding of this phenomenon.
\end{abstract}

Keywords: Education; Medicalization; Methylphenidate; Pharmacological cognitive enhancement; Intellectual doping.

\title{
Resumen
}

El uso de medicamentos, por personas saludables, para mejorar el funcionamiento del cerebro y mejorar el desempeño cognitivo puede ser llamado de mejoramiento cognitivo farmacológico o de doping intelectual. Uno de los medicamentos más utilizados en Brasil con esta finalidad es el Clorhidrato de Metilfenidato, comercializado con el nombre de Ritalina o Concerta. Este artículo tuvo como objetivo comprender, a través de una revisión de la literatura, cómo se ha utilizado el clorhidrato de metilfenidato en lo mejoramiento cognitivo farmacológico y la relación de este fenómeno con la medicalización social. Se sabe que estos medicamentos tienen como efecto inmediato el aumento de la concentración, mas a largo plazo pueden causar supresión del crecimiento, aumento de la presión sanguínea, episodios psicóticos, entre otros. Sin embargo, han sido utilizados por universitarios con la finalidad de aumentar su capacidad productiva y cumplir plazos y metas. Como esta práctica ha ganado cada vez más adeptos y el acceso a este medicamento es facilitado, se hace necesario profundizar el entendimiento de este fenómeno.

Palabras-clave: Educación; Medicalización; Metilfenidato; Mejoramiento cognitivo farmacológico; Mejoramiento cognitivo. 


\section{Introdução}

O uso de medicamentos, por pessoas saudáveis, para melhorar o funcionamento do cérebro e aprimorar o desempenho cognitivo pode ser chamado de aprimoramento cognitivo farmacológico ou de doping intelectual. A discussão sobre este tema tem recebido espaço na mídia, pois sabe-se que esta prática tem ganhado cada vez mais adeptos, em todas as faixas etárias e classes sociais. Um dos medicamentos mais utilizados no Brasil com esta finalidade é o Cloridrato de Metilfenidato, comercializado com o nome de Ritalina ou Concerta, e ele tem sido utilizado por universitários, empresários e profissionais da saúde, com a finalidade de aumentar sua capacidade produtiva e cumprir prazos e metas. Estes medicamentos tem como efeito imediato o aumento da concentração, mas ao longo prazo podem causar supressão do crescimento, aumento da pressão sanguínea, episódios psicóticos, entre outros.

Este fenômeno está dentro da lógica da medicalização, que segundo Lemos (2014), não se restringe à patologização, uma vez que também pode operar sobre a gestão preventiva da saúde, em nome do aumento da longevidade, do governo da saúde, da produção acelerada e ampliada nos estudos e no trabalho, de melhora das performances sexuais e físicas, de estimulação cognitiva com promessas de maior concentração em provas e concursos, etc. A autora ainda afirma que:

(...) vivemos em uma sociedade que não aceita o sofrimento, o choro, a tristeza, a crítica, a fala subversiva, a dissidência, e que reitera uma ilusão de felicidade, de alto desempenho e de saúde como salvação idealizada. (...) A intensidade cada vez maior de encomendas de competição no trabalho e de exigências altíssimas de desempenho por metas, somada ao esgarçamento da solidariedade e à fragmentação dos laços sociais, cria espaço para a ampliação da medicalização dos corpos. Situações que possam diminuir as capacidades e/ou torná-las mais lentas ou excessivamente rápidas, a ponto de prejudicar as performances, e qualquer movimento de resistência à lógica consumista e de mercado de cada ato cotidiano são rapidamente medicalizadas (...). (p. 492).

Neste contexto, o presente artigo objetivou compreender como o cloridrato metilfenidato tem sido utilizado no aprimoramento cognitivo farmacológico e a relação deste fenômeno com a medicalização social. É relevante estudar este tema em virtude da importância que ele tem tido na atualidade e do espaço que tem ganhado no contexto social, bem como porque através das discussões se buscará desmistificar a ideia que os problemas 
cotidianos podem ser resolvidos com pílulas e que o cérebro humano sempre poderia atingir níveis mais altos.

\section{Metodologia}

Para atingir o objetivo foi realizada uma pesquisa de revisão de literatura, com o intuito de explicitar como estas ideias estão sendo apropriadas por nossa sociedade.

A pesquisa de revisão de literatura consiste em uma avaliação crítica de materiais que já foram publicados, com o intuito de esclarecer um determinado problema, sumarizando estudos prévios e informando o estado em que se encontra a área de investigação, com vistas a aprofundar o conhecimento sobre o tema (Pereira et al, 2018).

O levantamento bibliográfico foi realizado por meio dos sites Scielo, PubMed, Psicoinfo e do banco de teses da Coordenação de Aperfeiçoamento de Pessoal de Nível Superior (CAPES), bem como em livros de bibliotecas e do acervo pessoal da autora. As palavras-chave utilizadas na busca foram aprimoramento cognitivo farmacológico, medicalização, doping intelectual, pharmacological cognitive enhancement, medicalization. Dos trabalhos encontrados foram selecionados os mais relevantes para a escrita deste artigo.

Para melhor organização o manuscrito apresentará inicialmente a relação entre a medicalização social e a medicalização escolar, partindo da definição destes constructos; posteriormente discutirá o aprimoramento cognitivo farmacológico ou doping intelectual e o medicamento utilizado para este fim; e na sequência serão apontadas as considerações finais a respeito do tema. É importante ressaltar que o mesmo não tem a pretensão de esgotar o assunto, mas tão somente pontuar algumas questões importantes para entendê-lo, visto que a seleção dos referenciais teóricos reflete as perspectivas da autora.

\section{Medicalização Escolar}

\subsection{A medicalização social}

Para melhor entendermos este tema precisamos inicialmente conceituar medicalização e medicamentalização. Dá-se o nome de medicalização ao processo por meio do qual são deslocados para o campo médico problemas que fazem parte do cotidiano dos indivíduos, que teriam origem social e política. A medicalização seria o processo que transforma sensações 
físicas ou psicológicas normais (como insônia e tristeza) em sintomas de doenças (como distúrbio do sono e depressão). Dentro desse processo há outro, denominado medicamentalização, que se refere ao uso excessivo de medicamentos para alívio de dores cotidianas. Estes dois processos podem coexistir ou não, havendo casos em que ocorre somente a medicalização, sem uso de medicamentos, e em outros casos há uso desenfreado de medicamentos sem que haja um diagnóstico médico. Os dois são preocupantes e estão muito difundidos na sociedade, e por isso, serão analisados detalhadamente.

Uma das primeiras pessoas a examinar a medicalização foi o filósofo Ivan Illich. Para ele, com o desenvolvimento da medicina diminuiu a autonomia dos indivíduos no seu cuidado com a saúde e aumentou o poder do médico. Com isso,

curar não é mais compreendido com a atividade do doente e se torna cada vez mais o ato daquele que se encarrega do paciente. Quando este terceiro surge e cobra por seus serviços, curar passa por uma primeira transformação: muda de dom para mercadoria. Quando o terapeuta se torna escolarizado, curar transforma-se de um simples serviço num míster profissional. Quando o sentido transitivo domina a linguagem, o funcionário provedor de cura obtém o monopólio. O que é abundante, gratuito e de grande valor torna-se alguma coisa que, por definição, é rara, tem um custo monetário de produção e um preço de mercado. Curar não é mais uma atividade, mas uma mercadoria (Illich, 1975, p. 78-79).

Assim, os rituais criados pela medicina teriam tornado a dor, a doença e a morte estímulos à produção de mercadorias, o que teria levado a indústria de cuidados médicos a crescer tanto. No entanto, o autor alerta que a medicina pode ser iatrogênica, ou seja, ela mesma, juntamente com os medicamentos e os hospitais, poderia produzir doenças. Isso poderia acontecer, por exemplo, por meio da iatrogênese social que está produzindo uma sociedade cada vez mais mórbida. Ou seja, o impacto social da medicina, mais do que sua ação técnica direta, estaria criando muitos sintomas clínicos, que não poderiam ser produzidos por intervenção isolada de um médico. Uma das vertentes dessa iatrogênese social é a medicalização do diagnóstico, que leva o indivíduo a tornar-se um paciente potencial, um objeto pelo qual o médico é responsável. Isso levaria a uma eliminação progressiva da saúde, graças à multiplicação ilimitada dos papeis de doente (Illich, 1975). Endossam esse cenário as novas categorizações médicas (CID - Código Internacional de Doenças e DSM- Manual Diagnóstico e Estatístico de Transtornos Mentais), que a cada ano aumentam o número dos que se enquadram na "anormalidade", tornando exceção os que não se encaixam em nenhuma categoria. 
As críticas do autor citado acima ainda são muito atuais, pois a cada dia esses conceitos tem se mostrado mais concretos. Atualmente é muito difundida a ideia de que o bem estar exige a eliminação da dor, a correção de todas as anomalias, o desaparecimento das doenças e a luta contra a morte. Tudo isso graças ao modelo biomédico que privilegia o caráter biológico do processo saúde-doença e define os sujeitos enquanto corpo biológico. Com isso as pessoas passam a se descrever por meio de uma linguagem somática pondo em segundo plano, variáveis de cunho psicológico e, econômico-social.

A biologização, embasada em concepção determinista, em que todos os aspectos da vida seriam determinados por estruturas biológicas que não interagiriam com $o$ ambiente, retira do cenário todos os processos e fenômenos característicos da vida em sociedade, como a historicidade, a cultura, a organização social com suas desigualdades de inserção e de acesso, valores, afetos, etc. Reduzida a vida a seu substrato biológico, de modo que todo futuro estaria irreversivelmente determinado desde o início, está preparado o terreno para a medicalização (...) (Collares \& Moysés, 2014, p. 51-52).

A medicalização leva a uma epidemia de diagnósticos, que por sua vez leva a uma epidemia de tratamentos e tem múltiplas causas. Entre elas estão o conjunto de transições e transformações que se operam em todo o mundo e em todos os sistemas de saúde. A primeira é a transição demográfica, com o rápido envelhecimento da população, redução da taxa de mortalidade em geral e da taxa de fertilidade, o que aponta para um horizonte em que a pirâmide demográfica muda rapidamente.

A segunda é a transição epidemiológica, onde doenças crônicas passam a ocupar um papel central na sociedade brasileira. A transição alimentar é consequência das transformações da família moderna e das tecnologias de produção de alimentos, com queda da qualidade do que se come. A transição tecnológica trouxe biodrogas, vacinas terapêuticas, tratamentos mais individualizados, medicina personalizada, e isso gera impacto nos custos e afeta a questão da equidade e da sustentabilidade dos sistemas de saúde. Por fim, a transição cultural trouxe o acesso a um grande número de informações, mas também uma grande disseminação do marketing comercial da indústria farmacêutica (Temporão, 2013). Essas transformações tiveram grandes implicações na vida das pessoas, pois as têm levado a acreditar que a medicina e os medicamentos poderiam resolver todos os problemas.

Neste novo cenário, segundo Guarido (2009), a produção dos remédios e seu uso, não podem mais ser vistos somente no campo científico e da prática médica, pois atualmente eles apresentam-se como novos bens a consumir, atrelados à condição de produção de bem-estar, 
felicidade e autorrealização, ideias do mundo contemporâneo. Essas "pílulas mágicas" têm sido usadas como “"amortecedores químicos” para as situações inerentes à vida como o luto, o estresse, o medo, a solidão, a tristeza ou os sentimentos de inferioridade." (Santos \& Farias, 2010, p. 268-269).

No entanto, os novos ideais impostos pela sociedade, podem nos levar ao adoecimento, pois quando se tem um conjunto de comportamentos esperados para uma pessoa, estabelecendo que ser normal e saudável é ser acima da média, tem-se, inevitavelmente, em contrapartida, pessoas que não vão atingir o que é estabelecido. Em relação ao contexto educacional, ser normal é ser atento, quieto, e ter sempre um bom rendimento. Quando isso não acontece é porque há alguma coisa errada, então é preciso investigar e tratar. Segundo o modelo biologizante e medicalizante, a razão do não aprender ou não se comportar estaria no cérebro das crianças. Os problemas neurológicos interfeririam em problemas considerados pré-requisitos para a aprendizagem, como percepção e processamento de informações, utilização de estratégias cognitivas, habilidades motoras, atenção, linguagem, raciocínio matemático, habilidades sociais etc (Meira, 2012).

Segundo esse discurso em relação a um aluno com dificuldades de leitura e escrita não se questionaria a escola, o método, as condições de aprendizagem e de escolarização, mas se buscaria na criança e no seu cérebro as causas dessas dificuldades. Como as causas do insucesso estão na criança, a "cura" para o insucesso também estaria nela, sendo necessária apenas a administração do medicamento correto com o intuito de reajustar a química cerebral para que tudo volte a funcionar adequadamente.

Com novas descobertas científicas aparecendo como explicação dos comportamentos, das sensações e do sofrimento humano, o mau funcionamento do cérebro passou a ser facilmente detectado e tratado para que funcione com perfeição. O que está ruim precisa ficar bom e que está bom sempre pode melhorar. Neste contexto, os achados neurocientíficos passam a ter grande importância para o ambiente escolar, pois eles podem contribuir para o aperfeiçoamento do processo educacional. Entre esses supostos achados científicos estão alguns medicamentos que estão sendo utilizados para auxiliar o funcionamento de um cérebro que, apesar de normal, estaria encontrando dificuldade para atingir os objetivos propostos, pois na corrida pelo sucesso ninguém quer ficar para trás. 


\subsection{O doping intelectual}

Com base nos conceitos anteriormente discutidos pode-se mais facilmente entender os que serão apresentados a seguir. Neste momento será focalizado o uso de medicamentos não destinados para fins terapêuticos, mas para fins cosméticos, recreativos e aprimoradores. $\mathrm{Ou}$ seja, nesses casos que serão apresentados o medicamento não teria a função de tratar ou curar uma doença, mas de melhorar o funcionamento de um organismo sadio para que ele atinja o máximo do seu potencial. Serão focados os medicamentos que poderiam melhorar as funções cognitivas.

O aprimoramento cognitivo farmacológico também é chamado de doping intelectual, neuroaprimoramento farmacológico, aperfeiçoamento cognitivo, uso instrumental de remédios, drogas para "turbinar" o cérebro, ampliadores cognitivos, drogas nootrópicas, neurologia cosmética, drogas da inteligência, Botox do cérebro, Viagra para o cérebro, anabolizante cerebral, droga de estudo, pílula da boa nota, e seus correspondentes em língua inglesa, neurocognitive enhancement, pharmacological cognitive enhancement, smart drugs, nootropic drugs, performance enhancing substances, central nervous system-stimulating drugs, good-grade pills, technologies of optimization e, se referem ao uso de novas tecnologias neurocientíficas para monitoramento e manipulação das funções cerebrais, por exemplo, usar remédios para melhorar o funcionamento do cérebro e aprimorar o desempenho cognitivo.

O interesse por estimulantes cognitivos surgiu no início dos anos 1990 com uma espécie de obsessão pública por produtos que pudessem aumentar artificialmente a inteligência, proporcionando um cérebro potente, que pensasse rápido, se lembrasse mais depressa das coisas, e com isso garantisse algumas vantagens competitivas. Isso está sendo feito por meio do uso de drogas capazes de melhorar a memória de pessoas com idade avançada, ou com algum tipo de demência, hiperatividade ou déficit de atenção, que está sendo apropriado por não doentes (Totti, 2008). Essa prática vem suscitando acirrado debate, inclusive ético, nos meios científicos e políticos.

Uma das áreas que estuda essa questão é a Bioética. Neste campo do conhecimento, discutir este tema é importante primeiro porque a dificuldade de diferenciar saúde e doença mental faz com que seja difícil estabelecer quem precisaria ou não de intervenção. Em segundo lugar, intervenções no cérebro têm implicações éticas diferentes de intervenções em outros órgãos (Farah \& Wolpe, 2004). 
As principais preocupações em relação ao neuroaprimoramento farmacológico se referem a três grandes temas: segurança, liberdade de escolha e igualdade de acesso. $\mathrm{O}$ tema da segurança diz respeito às consequências individuais dessa prática e envolve os métodos e tempos de exposição para avaliação dos efeitos colaterais, a ponderação entre riscos e benefícios e os efeitos da medicação em um órgão pouco conhecido como é o cérebro. Já o segundo e o terceiro temas estão relacionados à repercussão social do aprimoramento cognitivo farmacológico (Barros, 2009).

Sobre a liberdade de escolha há a contrapartida da coação. Farah (2002) acrescenta que além da coerção direta, existe o risco de uma coerção indireta, uma vez que é difícil preservar o direito de não usar o medicamento em uma sociedade competitiva como a sociedade ocidental. Com isso, a difusão do aprimoramento poderia aumentar os níveis de exigência, deixando em desvantagem as pessoas que não fizessem uso desse método de aprimoramento. Outro risco seria o uso dos medicamentos por adolescentes e adultos para praticar atividades triviais ou mesmo em atividades como jogos, ou seja, não haveria como garantir o uso adequado do fármaco. Já em relação ao acesso, possivelmente ele não estaria disponível para todos, o que intensificaria a desigualdade social. Teixeira (2007) resume estas ideias.

Se o aperfeiçoamento artificial das capacidades cognitivas se espalhar, as pessoas poderão vir a ser pressionadas para usar drogas aperfeiçoadoras. A pressão poderá ser explícita - por empregadores interessados em mais produtividade, por exemplo; ou mais sutil: se seu colega de classe usa estimuladores das funções cognitivas e você não, quem vai obter melhores notas e, possivelmente, encontrar as melhores posições disponíveis no mercado de trabalho? Por outro lado, pais desejosos de ver alto desempenho em seus filhos menores terão motivo e oportunidade para dar a eles drogas aperfeiçoadoras. Mais: em uma sociedade tão desigual, também o acesso a essas drogas poderá ser desigual. (p. 501).

Há também quem questione o aprimoramento cognitivo farmacológico como sendo uma fraude ou desonestidade. Isto seria fruto da comparação entre essa prática e o doping, ou seja, o uso de substâncias proibidas por atletas para melhor a sua performance. No entanto, alguns autores respondem que no caso dos esportes, por exemplo, determina-se que os atletas não podem usar substâncias específicas enquanto estiverem competindo. O entendimento que o doping esportivo é uma fraude/desonestidade resulta da ideia de que otimizar a performance por meio de medicamentos é uma maneira mais fácil de chegar ao objetivo, o que não 
ocorreria no caso do doping intelectual, já que os usuários ainda precisariam se esforçar para alcançar os objetivos (Barros, 2009).

Por outro lado, há autores que defendem a prática, como Greely et al. (2008) e, que acreditam que ela pode ser segura. Para isso propõem medidas como uma política de saúde formada por uma variada fonte de recursos científicos, profissionais, educacionais e sociais, somados à legislação. As sugestões incluem quatro mecanismos: o primeiro seria um programa de pesquisa sobre o uso e os impactos das drogas para aprimoramento cognitivo em indivíduos saudáveis, que produziria informações de qualidade sobre os riscos e benefícios do uso dos fármacos; o segundo mecanismo seria a participação de organizações profissionais para formular guias para que os profissionais saibam como lidar com essa prática para melhor encaminharem os usuários dos fármacos; o terceiro sugere que sejam divulgadas as informações sobre os riscos e benefícios, bem como as alternativas para o aprimoramento cognitivo farmacológico e, por fim, há a recomendação de que haja uma ação legislativa cuidadosa e limitada para tornar o método do neuroaprimoramento farmacológico um caminho útil.

Tudo isso deve ser feito porque a diferença classificatória entre o remédio e o veneno não estaria associada somente à dose, mas, também, à legitimidade institucional, o assimilando ou marginalizando. Quando uma droga psicoativa é legalmente considerada como terapêutica, a medicina torna-se a instituição responsável pelo estabelecimento do uso "controlado". Os usuários de uma mesma substância são reconhecidos socialmente como doentes quando recebem uma prescrição médica, e são rotulados como dependentes químicos na utilização para outros fins (Brant \& Carvalho, 2012).

Segundo Itaborahy (2009), o debate sobre o uso de medicamentos para fins de aprimoramento não está desconectado da atual prática de prescrição médica, nem dos mecanismos da indústria farmacêutica em investir em propagandas que vendem sucesso, ou do investimento em pesquisas que indicam frequentemente a ampliação de critérios diagnósticos, sempre aumentando seu público em potencial e seu "uso médico". No entanto, é preciso ter em mente que há formas de melhorar o desempenho cognitivo por outros meios, como atividades físicas, alimentação saudável, sono regular, assim como leituras e exercícios, que seriam alternativas que exigem mais esforço, mas são menos invasivas.

Um dos medicamentos mais utilizados no Brasil com a finalidade de melhorar o funcionamento do cérebro é o cloridrato de metilfenidato, vendido com os nomes de Ritalina e Ritalina LA (Novartis) e Concerta (Janssen-Cilag), e segundo a lista de medicamentos genéricos registrados na ANVISA, ainda não foi aprovado medicamento genérico com esta 
base farmacológica. O composto foi sintetizado por Leandro Panizzon, farmacêutico da antiga empresa CIBA (atualmente Novartis S/A) na Suíça, sendo patenteado em 1954. Foi comercializado inicialmente como um psicoestimulante leve. Chegou aos EUA em 1956 e ao Brasil em 1998. O nome Ritalina se deve ao apelido da esposa do Dr. Panizzon, Marguerite, chamada de Rita, o que levou a Ritaline. Derivado da anfetamina, esse estimulante era indicado nos anos 1950 para fadiga crônica e narcolepsia. A esposa do Dr. Panizzon utilizava Ritalina com frequência, por ter a pressão arterial muito baixa, e beneficiava-se de sua ação tonificante e estimulante tomando um comprimido antes de uma partida de tênis, por exemplo (Weber, 2000).

O metilfenidato era indicado inicialmente para tratamento da narcolepsia, um raro transtorno do sono. Contudo, seu uso logo foi ampliado. Nos anos 1960, a medicação já era considerada útil para a maioria dos quadros psiquiátricos, incluindo casos de pacientes em psicoterapia e em tratamento com outras drogas mais potentes. A partir dos anos 1960, estudos começaram a ressaltar os benefícios do metilfenidato para tratamento de crianças hiperativas e distraídas, e hoje sua principal indicação terapêutica é para o tratamento de Transtorno de Déficit de Atenção e Hiperatividade (TDAH) em crianças (Barros, 2009).

Atualmente este medicamento também tem sido usado para potencializar performances escolares e laborais. Elevados níveis de produtividade em curto prazo, com baixo custo e alta qualidade, são imperativos colocados pela cultura do sucesso a qualquer custo. “A produção do ideal de ser protagonista em cenários de sucesso está articulada ao discurso de que tudo depende, exclusivamente, da força de vontade do sujeito" (Brant \& Carvalho, 2012, p. 624). Para isso, pessoas reconhecidamente saudáveis estão utilizando o fármaco como gadget, uma mercadoria, um verdadeiro acessório para a obtenção da exacerbação produtiva, como afirmam os autores.

Esse uso não terapêutico se faz, em grande parte, por universitários, empresários e profissionais da área de saúde, que, em geral, têm maior conhecimento sobre a droga do que o restante da população. Este público, que utiliza o fármaco para aprimoramento, recreação ou para emagrecimento, também tem feito uso do mesmo de forma triturada como pó ou diluído em água para ser injetado (ANVISA, 2010).

A bula do medicamento alerta para o risco da dependência, física e psíquica. A dependência psicológica pode inclusive levar o usuário a acreditar não mais ser capaz de produzir sem o uso do medicamento. Além disso, o aumento da capacidade produtiva seria resultado de processos neuroquímicos "turbinados pela substância” (Brant \& Carvalho, 2012, p. 629). Assim, pode-se questionar: "Chegará o dia em que se pedirá aos estudantes que 
tenham à mão uma amostra de urina, para ser entregue junto com as provas escolares, para provar que seus resultados são o resultado de trabalho duro e não de farmacologia?" (Butcher, 2003, p. 132). Outra questão a ser levantada é sobre a duração do tratamento com o metilfenidato e seus efeitos no organismo em longo prazo, pois somente sua eficácia em curto prazo é conhecida.

Devido à sua importância, a discussão sobre esse tema tem recebido espaço nos meios científicos, pois se sabe que essa prática tem ganhado cada vez mais adeptos em todas as faixas etárias e classes sociais. No Brasil, o consumo do metilfenidato para fins de aprimoramento foi encontrado com uma prevalência de $0,9 \%$ em estudantes de universidades públicas e privadas das 27 capitais brasileiras (Cesar et al., 2012); 9,4\% em estudantes de medicina de uma universidade do Sul de Minas Gerais (Silveira et al., 2015); 18,75\% em estudantes de diversos cursos de uma faculdade de Curitiba (PR) (Tsuda \& Christoff, 2015); 60\% em universitários dos cursos de farmácia e medicina de Campos dos Goytacazes (RJ) (Mota \& Pessanha, 2014). Em relação ao público pré-universitário a pesquisa de Cassimiro (2012) mostrou que 15\% dos alunos dos dois cursos pré-vestibulares de Belo Horizonte (MG) pesquisados por ele fazia uso do cloridrato de metilfenidato. Não houve diferença estatisticamente significativa entre as prevalências do uso de psicofármacos entre os alunos de diferentes áreas de escolha para o vestibular, mas separando por área de interesse, verificou-se que a maioria dos usuários desejava ingressar em medicina.

A maioria dos estudos apresentados acima foi desenvolvida com universitários, particularmente estudantes de medicina. Este fato pode estar relacionado ao acesso facilitado dos pesquisadores a este público, mas também pode ser devido a um uso maior por esses estudantes em virtude da sobrecarga do vasto conteúdo abordado, bem como pelos momentos de estresse durante o curso. Além disso, o contato com a doença e a morte, a competição profissional e o receio de não conseguir satisfazer as exigências do curso podem intensificar sentimentos de angústia e estresse entre eles, levando-os ao consumo de substâncias psicoativas (Bassols et al., 2008).

A pesquisa de Barros (2009) com estudantes universitários brasileiros buscou investigar a representação social dos mesmos sobre o aprimoramento cognitivo com o uso do metilfenidato. A maioria dos estudantes entrevistados não tinha conhecimento sobre o uso deste medicamento para facilitar o estudo, mas acreditavam que entre os motivos que levam uma pessoa a fazer uso dele estão a pressão social para melhorar o desempenho e ter um comportamento sobre-humano, além do limite, e uma postura competitiva, tanto com os outros como consigo mesmo. Segundo os alunos, seria importante ter um excelente 
desempenho profissional, pois este seria o principal meio de inserção no grupo. No entanto, foi expressa a preocupação com o risco de haver uma coerção social direta e indireta para uso do remédio, e com a possibilidade de o aprimoramento cognitivo farmacológico aumentar a injustiça social, caso haja um acesso desigual a essa prática.

Andrade e colaboradores (2011) apontam que médicos brasileiros também percebem essa prática como sendo controversa, pois envolve aspectos éticos, sociais e legais. A maioria deles afirmou que não prescreveria metilfenidato para fins de aprimoramento cognitivo, mas foram encontradas também respostas positivas, justificando que essa prática deve ser feita por um médico capacitado e com acompanhamento regular. Greely e colaboradores (2008) afirmam que médicos que veem a medicina voltada para a cura avaliarão o aprimoramento como inapropriado, enquanto aqueles que veem a medicina mais amplamente como um meio de ajudar os pacientes a viverem melhor e alcançarem seus objetivos poderão considerar o seu pedido de prescrição do medicamento. A reflexão de Carvalho, Brant e Melo (2014) segue a mesma direção, visto que afirmam que os médicos podem ter dificuldade em perceber os riscos contidos nessa demanda, uma vez que eles também convivem com pressões semelhantes às de seus pacientes e usuários, dada a azáfama entre plantões, estudos de caso e pesquisas.

Várias pesquisas confirmam uma crença favorável ao uso de aprimoradores cognitivos farmacológicos para conseguir realizar atividades cotidianas. Urrego e colaboradores (2008), por exemplo, identificaram que estudantes universitários utilizam estes medicamentos devido ao grande número de atividades acadêmicas, e Mota e Pessanha (2014) apontaram que a maioria usa nas vésperas das provas e para melhorar o rendimento na sala de aula e nos estágios. Tsuda e Christoff (2015) também apontaram como possíveis motivos as amizades e o ambiente, que levariam ao uso com o intuito de facilitar a comunicação, necessidade de obter mais diversão e obter sensações prazerosas, alívio do cansaço e estresse, e a pressão social com cobranças por parte da faculdade, do trabalho, dos familiares e amigos. Ram, Hussainy, Henning, Jensen e Russell (2016) ainda citam entre as razões para o uso do estimulante ajudar a relaxar e ser mais confiante. A pesquisa de Tsuda e Christoff (2015) indicou que os alunos admitiram o uso em consequência do calendário letivo, estabelecendo uma clara relação com as atividades acadêmicas, e tanto Barón et al (2011) quanto Carvalho (2016) verificaram um maior índice de consumo nos últimos semestres, o que pode estar relacionado ao aumento das exigências no decorrer do curso.

Segundo Eslami et al (2014) os universitários participantes de sua pesquisa afirmaram que o uso do metilfenidato era comum entre seus amigos e que eles aceitavam bem esse uso, e 
os universitários entrevistados por Barros (2009) também apontaram que a possibilidade do aprimoramento cognitivo não fere a sua moralidade, e que a prática não chegou a ser vista como algo repulsivo ou imoral a ser combatido.

Carvalho (2016) assinalou que dos 220 estudantes universitários que relataram fazer uso de substâncias psicoestimulantes, 80,9\% tiveram os efeitos esperados, entre eles o aumento da vigília foi o mais citado seguido de melhor rendimento nas atividades acadêmicas, aumento da concentração e maior capacidade intelectual. Mas Mota e Pessanha (2014) alertam que entre os usuários da sua amostra 86,6\% afirmaram já terem apresentado algum tipo de efeito colateral relatado na bula do medicamento. No entanto, mesmo apresentando efeitos colaterais eles continuaram utilizando, e entre os que não estão utilizando $65,7 \%$ relataram que é devido à dificuldade de encontrar o fármaco no mercado e por doenças, e só 34,3\% devido aos efeitos colaterais. Carneiro et al (2013) também alertaram que 51,35\% dos usuários afirmaram sentir-se cansados após o uso, e 10,81\% tiveram que aumentar a dose para obter o mesmo efeito, e mesmo com efeitos colaterais $27,03 \%$ continuaram fazendo uso.

Mota e Pessanha (2014) também observaram que 57,7\% dos universitários pesquisados afirmaram que ao término do curso pretendem continuar utilizando o metilfenidato e os principais objetivos para isso seriam concurso público, residência, mestrado/doutorado e entrevista de emprego. Emanuel et al (2013) ao perguntar aos universitários se suas experiências com o medicamento poderiam impactar sua decisão de prescrevê-lo a seus pacientes $16 \%$ disseram que seriam mais propensos a prescrever, $26 \%$ seriam menos propensos a prescrever e $61 \%$ se sentiam inseguros para responder. Quando perguntados se eles se imaginariam usando o estimulantes dali a 5 anos $6 \%$ afirmou que provavelmente ou definitivamente não, $17 \%$ provavelmente ou definitivamente sim, $15 \%$ estavam em dúvida.

Vale ressaltar que os efeitos do fármaco no organismo ainda não foram completamente identificados, havendo entre os pesquisadores muitas controvérsias. Seus efeitos na memória seriam em áreas específicas, como, por exemplo, na memória de trabalho, que seria responsável pela evocação das informações que estão em uso no momento, permitindo processos cognitivos como compreensão e pensamento lógico (Freese, Signor, Machado, Ferigolo, \& Barros, 2012). Nascimento e Bastos (2011) apontam que o fármaco em dose única seria capaz de melhorar a memória, mas não a atenção, o humor, a vigília, a motivação e as funções executivas. Batistela, Bueno, Vaz e Galduróz (2016) apontam que não houve diferença no desempenho de sujeitos submetidos a testes de atenção, memória operacional e 
episódica, recebendo doses diferentes de metilfenidato (10, 20 e $40 \mathrm{mg}$ e placebo), tendo apenas um efeito na sensação subjetiva de bem-estar naqueles que receberam $40 \mathrm{mg}$. Vrecko (2013) afirma que o efeito do medicamento é mais emocional do que real, pois, afetando a capacidade de melhor conduzir o trabalho, aumentaria em contrapartida a sensação de estresse e tensão.

Farah, Haimm, Sankoorikal e Chatterjee (2009) também citam um efeito negativo na criatividade, e Schaaf, Fallon, Huurne, Buitelaar e Cools (2013) alertam que pode haver uma grande variação de resposta, tanto interindividual quanto intraindividual, o que faz com os que os resultados sejam muito diferentes. Já Machado e Toma (2016), em uma revisão sistemática, apontam que o efeito do metilfenidato em estudantes foi subjetivo, não melhorando o processo de aprendizagem e memória, apenas melhorando a atenção na hora de estudar. Isso equivaleria, por exemplo, a ter um bom desempenho durante a fase dos estudos e nenhuma melhora durante a realização das provas. No entanto, essa melhora sugestiva da atenção poderia levar a um aumento indiscriminado no consumo do medicamento. Urban e Gao (2014) também alertam que o uso indiscriminado antes dos 30 anos pode afetar a maturação do córtex pré-frontal, que é o local executivo da memória operacional, afetando o centro de controle de julgamentos, inibição de comportamentos e emoções.

Em resumo, os efeitos do metilfenidato em relação ao aprimoramento cognitivo ainda não são conclusivos. Ele pode, em geral, influenciar o desempenho como resultado de seu efeito estimulante, no entanto, as evidências não apoiam a conclusão de que ele possa promover um melhor desempenho cognitivo, principalmente se usado sem prescrição médica, em doses excessivas, ou de forma inadequada, como por administração nasal ou intravenosa.

\section{Considerações Finais}

Segundo a definição do Fórum sobre Medicalização da Educação e da Sociedade, publicada na Carta do IV Seminário Internacional A educação medicalizada: desver o mundo, perturbar os sentidos (2015), a medicalização envolve um tipo de racionalidade determinista que desconsidera a complexidade da vida humana, a reduzindo a questões de cunho individual, seja em seu aspecto orgânico, seja em seu aspecto psíquico, seja em uma leitura restrita e naturalizada dos aspectos sociais. Nessa concepção, características comportamentais são tomadas apenas a partir da perspectiva do indivíduo isolado, que passaria a ser o único responsável por sua inadaptação às normas e padrões sociais dominantes, sendo esse fenômeno mais amplo do que a prescrição (abusiva ou não) de medicamentos. 
Neste contexto, ao entender que tudo aquilo que é capaz de gerar um mínimo de desconforto e mal-estar pode ser explicado através dos níveis de determinados neurotransmissores e passível de ser medicado, o discurso psicofarmacológico coloca a questão do medicamento em uma posição central e preocupante em nossa sociedade. Isso porque, segundo Canabarro e Alves (2009), ao longo da história da civilização, as substâncias psicoativas não foram usadas somente no tratamento das afecções mentais e como parte de rituais religiosos. Pelo contrário, em diferentes momentos da história o homem procurou essas substâncias buscando tornar-se insensível aos infortúnios da vida.

Atualmente os medicamentos têm sido usados também para melhorar performances físicas como ter um corpo mais adaptado, reduzir peso, reduzir calvície, melhorar comportamentos indesejáveis ou para ter um melhor desempenho em atividades esportivas, sociais ou cognitivas. Este último refere-se ao denominado de aprimoramento cognitivo farmacológico, que diz respeito ao uso de medicamentos para manipular e melhorar as funções cerebrais.

A partir de todas as informações apresentadas neste texto pode-se concluir que esta prática é disseminada, visto que os estudos aqui citados mostraram dados de diversas localidades. Por isso, considerando o alto potencial de abuso e dependência do medicamento torna-se urgente a realização de discussões que abordem a atual problemática do consumo indevido, alertando a população para o mau uso, os efeitos adversos já descritos e os ainda desconhecidos. Assim, considerando que a sociedade credita grande valor ao sucesso e bom desempenho pessoal, mas considerando também toda a complexidade do sistema educacional brasileiro, se torna imprescindível discutir a medicalização escolar que está hoje em evidência, onde os medicamentos são vistos por vários estudantes como objetos de desejo e consumo. Uma mudança nessa percepção deve ser feita com a participação de todos os setores da sociedade, a fim de propor medidas preventivas e/ou de oferecer apoio para os estudantes, com vistas a proporcionar uma melhor formação profissional sem riscos para a saúde.

Por fim conclui-se que a presente pesquisa de revisão de literatura mostrou-se importante tendo em vista que análises relacionadas ao aprimoramento cognitivo farmacológico não são frequentes, sendo necessária a realização de estudos empíricos para entender melhor o que os jovens pensam sobre o assunto, e com isso contribuir com o avanço do conhecimento. 


\section{Referências}

Agência Nacional de Vigilância Sanitária- ANVISA. (2010). Sistema Nacional de Gerenciamento de Produtos Controlados: resultados 2009. Brasília: Agência Nacional de Vigilância Sanitária. Recuperado de: http://www.anvisa.gov.br/sngpc/resultados_2009.pdf>.

Agência Nacional de Vigilância Sanitária- ANVISA. (n.d). Lista de medicamentos genéricos registrados na Anvisa. Recuperado de: http://portal.anvisa.gov.br/medicamentos-genericosregistrados Aceso em 01 de maio de 2017

Andrade, DC, Lima, LRO, Saldanha, LVR, Souza, MM, Simões, VS, Barreto, WWP \& Morato, MJF. (2011). Uso do metilfenidato para além do transtorno de déficit de atenção e hiperatividade. (Trabalho de Conclusão de Curso). Universidade Vale do Rio Doce, Faculdade de Ciências da Saúde, Governador Valadares- MG.

Barros, DB. (2009). Aprimoramento cognitivo farmacológico: grupos focais com universitários. Dissertação de mestrado. Instituto de Medicina Social, Universidade do Estado do Rio de Janeiro, Rio de Janeiro.

Bassols, AM, Sordi, AO, Eizirik, CL, Seeger, GM, Rodrigues, GS \& Reche, M. (2008). A prevalência de estresse em uma amostra de estudantes do curso de medicina da Universidade Federal do Rio Grande do Sul. Revista HCPA. 28 (3), 153-7.

Barón, L, Botero-Henao, K, Castaño-Castrillón, JJ, Castillo-Chang, K, Díaz-Corrales, J, Echeverri-Uribe, JS, Parra, GA, Yepes, JS \& Yurgaky-Jordán, HY. (2011). Prevalencia y factores asociados al consumo de anfetaminas, en estudiantes del programa de medicina de la universidad de Manizales (Colombia), 2010. Rev.fac.med. 59 (3), 201-214.

Batistela, S, Bueno, OFA, Vaz, LJ \& Galduróz, JCF. (2016). Methylphenidate as a cognitive enhancer in healthy young people. Dementia \& Neuropsychologia, 10 (2), 134-142.

Brant, LC \& Carvalho, TRF. ( 2012). Methylphenidate: medication as a "gadget" of contemporary life. Interface - Comunic., Saude, Educ., 16 (42), 623-36. 
Butcher, J. (2003). Cognitive enhancement raises ethical concerns. Lancet, 362 (9078), 132133.

Canabarro, RCS \& Alves, MB. (2009). Uma pílula para (não) viver. Revista mal-estar e subjetividade, 9(3), 839-866.

Carneiro, SG, Prado, AST, Moura, HC, Strapasson, JF, Rabelo, NF, Ribeiro, TT, \& Jesus, EC. (2013). O uso não prescrito de metilfenidato entre acadêmicos de medicina. Cadernos UniFOA, 1 (1), 53-59.

Carvalho, AHM. (2016). Avaliação do uso de substâncias psicoestimulantes entre universitários para melhoria do desempenho acadêmico. (Trabalho de conclusão de curso Graduação). Curso de Farmácia, Centro Universitário Luterano de Palmas, Palmas-TO.

Carvalho, TRF, Brant, LC \& Melo, MB. (2014). Exigências de produtividade na escola e no trabalho e o consumo de metilfenidato. Educação \& Sociedade, 35 (127), 587-604.

Cassimiro, EE. (2012). Frequência do uso de psicofármacos entre jovens estudantes que cursam pré-vestibular. Adolesc. Saude, 9 (4), 27-36.

Cesar, ELR, Wagner, GA, Castaldelli- Maia, JM, Silveira, CM, Andrade, AG \& Oliveira, LG. (2012). Uso prescrito de cloridrato de metilfenidato e correlatos entre estudantes universitários brasileiros. Revista de Psiquiatria Clínica. 39 (6), 183-188.

Collares, CAL \& Moysés, MAA. (2014). A educação na era dos transtornos. In Viégas, LS, Ribeiro, MIS, Oliveira, EC \& Teles, LAL. (Org). Medicalização da educação e da sociedade: Ciência ou mito? Salvador: EDUFBA.

Emanuel, RM, Frellsen, SL, Kashima, KJ, Sanquino, SM, Sierles, FS \& Lazarus, CJ (2013). Cognitive Enhancement Drug Use Among Future Physicians: Findings from a MultiInstitutional Census of Medical Students. J Gen Intern Med, 28 (8), 1028-1034. 
Eslami, AA, Jalilian, F, Ataee, M, Alavijeh, MM, Mahboubi, M, Afsar, A \& Aghaei, A. (2014). Intention and willingness in understanding Ritalin misuse among Iranian medical college students: a cross-sectional study. Glob J Health Sci., 30 (6), 43-53.

Farah, MJ. (2002). Emerging ethical issues in neuroscience. Nature Neuroscience, 5 (11), 1123-1129.

Farah, MJ \& Wolpe, PR. (2004). Monitoring and Manipulating Brain Function: New Neuroscience Technologies and Their Ethical Implications. Hastings Center Report, 34 (3), $35-45$.

Farah, MJ, Haimm, C, Sankoorikal, G \& Chatterjee, A. (2009). When we enhance cognition with Adderall, do we sacrifice creativity? A preliminary study. Psychopharmacology, 202, $541-547$.

Fórum sobre medicalização da educação e da sociedade. (2015). Carta do IV Seminário Internacional a Educação Medicalizada: desver o mundo, perturbar os sentidos. SalvadorBA. Recuperado de: <http://seminario4.medicalizacao.org.br/carta-do-iv-seminariointernacional-a-educacao-medicalizada-desver-o-mundo-perturbar-ossentidos/> .

Freese, L, Signor, L, Machado, C, Ferigolo, M \& Barros HMT. (2012). Non-medical use of methylphenidate: a review. Trends Psychiatry Psychother, 34 (2), 110-5.

Greely, H et al. (2008). Towards responsible use of cognitive-enhancing drugs by the healthy. Nature, 456, 702-705.

Guarido, R. (2009). O sujeito refém do orgânico. Formação de Profissionais e a CriançaSujeito. Ano 7 col. LEPSI IP/FE-USP.

Illich, I. (1975). A expropriação da saúde: nêmesis da medicina. Rio de janeiro: Editora Nova Fronteira. 
Itaborahy, C. (2009). A Ritalina no Brasil: uma década de produção, divulgação e consumo. Dissertação de mestrado. Instituto de Medicina Social, Universidade do Estado do Rio de Janeiro, Rio de Janeiro.

Lemos, FCS. (2014). A medicalização da educação e da resistência no presente: disciplina, biopolítica e segurança. Psicol. Esc. Educ., 18 (3), 485-492.

Machado, LC \& Toma, MA. (2016). Qual a verdadeira função do metilfenidato na memória de indivíduos saudáveis? Revista UNILUS Ensino e Pesquisa. 13 (20), 126- 130.

Meira, MEM. (2012). Para uma crítica da medicalização na educação. Revista Semestral da Associação Brasileira de Psicologia Escolar e Educacional, 16 (1) 135-142.

Mota, JS \& Pessanha, FF. (2014). Prevalência do uso de metilfenidato por universitários de Campos dos Goytacazes, RJ. VÉRTICES, 6 (1), 77-86.

Nascimento, JLM \& Bastos, GNT. (2011) Psicofármacos para aprimoramento das funções cognitivas. II Jornada Fluminense de cognição Imune e Neural, Rio de Janeiro-RJ.

Pereira, AS et al. (2018). Metodologia da pesquisa científica. [e-book]. Santa Maria. Ed. UAB/NTE/UFSM. Disponível em:

https://repositorio.ufsm.br/bitstream/handle/1/15824/Lic_Computacao_MetodologiaPesquisa-Cientifica.pdf?sequence $=1$.

Ram, SS, Hussainy, S, Henning, M, Jensen, M \& Russell, B. (2016). Prevalence of cognitive enhancer use among New Zealand tertiary students. Drug Alcohol Rev, 35 (3), 345-451.

Ritalina®. (n.d.). Bula. Novartis Biociências S/A. Recuperado de: www.bulas.med.br/index Acesso em 01 de maio de 2017.

Santos, RI \& Farias, MR. (2010). Conflitos bioéticos e as políticas para acesso aos medicamentos. In: Caponi, S, Verdi, M, Brzozowski, FS \& Hellmann, F. (Org.). Medicalização da vida: ética, saúde pública e indústria farmacêutica. Palhoça: Ed. Unisul. 
Schaaf, ME, Fallon, SJ, Huurne, N, Buitelaar, J \& Cools, R. (2013). Working Memory

Capacity Predicts Effects of Methylphenidate on Reversal Learning.

Neuropsychopharmacology, 38, 2011-2018.

Silveira, VI, Oliveira, RJF, Caixeta, MR, Andrade, BBP, Siqueira, RGL \& Santos, GB.

(2015). Uso de psicoestimulantes por acadêmicos de medicina de uma universidade do sul de Minas Gerais. Revista da Universidade Vale do Rio Verde, 13 (2), 186-192.

Teixeira, M. (2007). Notícia preliminar sobre uma tendência contemporânea: o "aperfeiçoamento cognitivo" do ponto de vista da pesquisa em neurociências. Revista Latinoamericana de psicopatologia fundamental, 10 (3).

Temporão, JG. (2013). Indústria farmacêutica e medicalização. In Collares, CAL, Moysés, MAA \& Ribeiro, MCF. (Org). Novas capturas, antigos diagnósticos na era dos transtornos. Campinas - SP: Mercado de Letras.

Totti, BC. (2008). Notas preliminares sobre as drogas da inteligência. VII ESOCITE. Rio de Janeiro.

Tsuda, CA \& Christoff, AO. (2015). Avaliação do padrão de uso de estimulantes em uma faculdade de Curitiba - PR. Caderno da escola de saúde, 1 (13), 116- 132.

Urban, KR \& Gao, W. (2014). Performance enhancement at the cost of potential brain plasticity: neural ramifications of nootropic drugs in the healthy developing brain. Front. Syst. Neurosci, 8 (38), 1-10.

Urrego, MA et al. (2008). Consumo de anfetaminas, para mejorar rendimiento académico, en estudiantes de la Universidad de Manizales. Arch. Med, 9 (1), 43-57.

Vrecko, S. (2013). Just How Cognitive Is "Cognitive Enhancement"? On the Significance of Emotions in University Students' Experiences with Study Drugs. AJOB Neuroscience, 4 (1), $4-12$.

Weber, R. (2000). L'histoire de Ritalin. Life Sciences, 2, 8-9. 
Research, Society and Development, v. 9, n. 7, e379974301, 2020

(CC BY 4.0) | ISSN 2525-3409 | DOI: http://dx.doi.org/10.33448/rsd-v9i7.4301

Porcentagem de contribuição de cada autor no manuscrito

Emilia Suitberta de Oliveira Trigueiro - 100\% 